\title{
Luminal Vasopressin Modulates Transport in the Rabbit Cortical Collecting Duct
}

Yasuhiro Ando,* Kaoru Tabei, and Yasushi Asano

Division of Nephrology, Jichi Medical School Hospital, Tochigi 329-04, Japan

\begin{abstract}
We explored the action of luminal $A V P$ in rabbit $C C D$ perfused in vitro at $37^{\circ} \mathrm{C}$. Nanomolar concentrations of luminal AVP induced a sustained hyperpolarization of transepithelial voltage $(V)$ in contrast to a transient hyperpolarization caused by basolateral AVP. $10 \mu \mathrm{M}$ basolateral ouabain abolished the latter but not the former change in $V_{t}$. Despite a sustained hyperpolarization (from $-20.7 \pm 2.9$ to $-34.1 \pm 4.7 \mathrm{mV} ; P<0.01$ ), $10 \mathrm{nM}$ luminal AVP only slightly altered net $\mathrm{Na}^{+}$and $\mathrm{K}^{+}$fluxes $(7.6 \%$ stimulation and no significant change, respectively). Instead, luminal AVP appeared to modulate an acetazolamide-sensitive electrogenic ion transport because $200 \mu \mathrm{M}$ basolateral acetazolamide suppressed the luminal AVP-induced hyperpolarization (percentage of $V_{t}$ from $-50.4 \pm 10.8$ to $-5.1 \pm 1.4 ; P<0.005$ ). In terms of water transport, $10 \mathrm{nM}$ luminal AVP did not change hydraulic conductivity $\left(L_{\mathrm{p}}, \times 10^{-7} \mathrm{~cm} / \mathrm{atm}\right.$ per $\left.\mathrm{s}\right)($ from $3.9 \pm 0.8$ to 5.0 \pm 1.2 ), but suppressed the increase in $L_{\mathrm{p}}$ induced by 20 pM basolateral AVP (134.9 \pm 19.2 vs. 204.3 \pm 21.1 in control; $P$ $<0.05)$. These findings demonstrate distinct luminal action of AVP, suggesting amphilateral regulation of epithelial transport by AVP in the CCD. (J. Clin. Invest. 1991. 88:952-959.) Key words: water transport $\bullet$ transepithelial voltage $\bullet$ sodium reabsorption • potassium secretion • proton secretion
\end{abstract}

\section{Introduction}

In the mammalian kidney, the CCD is an important target in the nephron for the antidiuretic action of AVP, the single most important hormone for regulation of urine production. In this nephron segment, it has become evident that, in addition to the adenylyl cyclase-cyclic AMP system, which mediates the classic $V_{2}$ receptor-coupled antidiuretic effect of picomolar AVP, multiple signaling pathways are activated when AVP at nanomolar concentration is applied to either isolated tubules (1) or cells (2) in vitro. Unlike the cyclic AMP system, these additional signalings do not induce a hydroosmotic effect $(3,4)$, but result in self inhibition of the AVP-induced antidiuretic effect in the CCD (1). Also, these signalings may affect transport of ions such as $\mathrm{Na}^{+}, \mathrm{K}^{+}$, and $\mathrm{HCO}_{3}^{-}(5-7)$. However, the physiological relevance of these heterogeneous actions of nanomolar

Parts of this paper were presented at the 23rd annual meeting of the American Society of Nephrology in Washington, DC, December, 1990.

Address correspondence and reprint requests to Dr. Yasuhiro Ando, Division of Nephrology, Jichi Medical School Hospital, Tochigi 329-04, Japan.

Received for publication 19 December 1990 and in revised form 22 April 1991

J. Clin. Invest.

(c) The American Society for Clinical Investigation, Inc.

0021-9738/91/09/0952/08 \$2.00

Volume 88, September 1991, 952-959
AVP has not been confirmed, because the level of circulating AVP is strictly regulated within the picomolar range, and rarely exceeds this even under serious hypovolemia (8).

On the other hand, it is known that AVP is excreted into urine mainly via glomerular filtration and tubular excretion in the distal nephron (9-11). Importantly, when AVP release is stimulated, the final urine contains intact AVP of the nanomolar range, while plasma AVP level is within the picomolar range (9). Thus, in clarifying the comprehensive role of AVP in urine production, it is of interest to explore whether luminal AVP modulates the epithelial functions of the CCD.

Early in vitro microperfusion studies of the $\mathrm{CCD}$ by Grantham and Burg (12) revealed that luminal AVP $(\sim 500$ pM) failed to increase osmotic water permeability. In the amphibian urinary bladder epithelum, an analogue of mammalian collecting duct, AVP had also found not to increase water permeability (13) or to alter $\mathrm{Na}$ transport (14) when it was applied from the apical side. In a study using primary-cultured canine CCD cells (15), luminal AVP was found to stimulate prostaglandin synthesis. However, it is unknown whether cultured cells retain the intact apico-basolateral polarity of the $\mathrm{CCD}$ in situ. In fact, the luminal actions of bradykinin or prostaglandins found in the cultured CCD cells were not observed in isolated perfused CCDs $(6,16-19)$. Otherwise, to our knowledge, there are no published data describing an effect of luminal AVP. Given the high urinary AVP concentration and the multipotential of nanomolar AVP mentioned above, this study was undertaken to determine whether AVP has a luminal action other than increasing osmotic water permeability.

\section{Methods}

Single CCDs were dissected from kidneys of anesthetized (pentobarbital, intravenously injected) male Japanese white rabbits weighing 1.5$2.5 \mathrm{~kg}$, and perfused in a lucite bath chamber on the stage of an inverted microscope at $37^{\circ} \mathrm{C}$ using the methods described previously (20). To facilitate luminal perfusate exchange during each experiment, a polyethylene tube (PE-10; Clay Adams, Parsippany, NJ) was inserted in the "B" pipette, which was connected to the CCD lumen for perfusion. The perfusate flow rate was adjusted by hydrostatic pressure. Perfusate exchange was done manually by injecting the perfusate into the polyethylene tube, washing out the preexisting medium in the " $B$ " pipette.

The compositions of the bath medium and isotonic perfusate were as follows (in millimolars): $\mathrm{NaCl}, 105 ; \mathrm{NaHCO}_{3}, 25 ; \mathrm{Na}$ acetate, 10; $\mathrm{Na}_{2} \mathrm{HPO}_{4}, 2.3 ; \mathrm{NaH}_{2} \mathrm{PO}_{4}, 1.0 ; \mathrm{KCl}, 5 ; \mathrm{CaCl}_{2}, 1.8 ; \mathrm{MgSO}_{4}, 1.0 ;$ glucose, 8.3 ; alanine, 5 (osmolality $300 \mathrm{mOsmol}$ ). The composition of hypotonic perfusate was identical to that of the isotonic perfusate except for a lower osmolality ( $150 \mathrm{mOsmol})$ due to a lower $\mathrm{NaCl}$ concentration $(30 \mathrm{mM})$. Tritium-labeled inulin was added to the hypotonic perfusate as a volume marker. Both the isotonic and hypotonic perfusates contained $0.2 \mathrm{mg} / \mathrm{ml}$ food, drug, and cosmetic (FD\&C) dye for detection of cell damage and perfusate leakage. Before use, all solutions were bubbled to equilibration at $37^{\circ} \mathrm{C}$ with a $95 \% \mathrm{O}_{2} / 5 \% \mathrm{CO}_{2}$ gas mixture to achieve a pH of about 7.40, and a $\mathrm{PCO}_{2}$ of $\sim 40$ torr. To keep $\mathrm{PCO}_{2}$ and $\mathrm{pH}$ constant, the bath medium was held in a $60 \mathrm{ml}$ plastic syringe connected to the bath chamber by a polyethylene tube (PE-100; Clay Adams) and exchanged continuously during the experiment at a flow 
rate of $25 \mathrm{ml} / \mathrm{h}$ by using a syringe pump (model STC-521; Terumo Inc., Tokyo). Perfusates were also stored in plastic syringes until use. The pH values of the bath medium and perfusate were checked again each time just before use using a pH meter (model M-220; Corning Glass Inc., Corning, NY). Experiments were started after the tubules had been perfused for $50-90 \mathrm{~min}$ at $37^{\circ} \mathrm{C}$ to obtain a stable transepithelial voltage $\left(V_{t}\right)$ and to eliminate any residual actions of endogenous AVP (1).

Measurement of transepithelial voltage $(V)^{1}(\mathrm{mV})$ : voltage between two calomel cell electrodes, each connected by a Ringer-agarose bridge to the bath medium and the perfusate in the " $\mathrm{B}$ " pipette, was monitored continuously by an electrometer (model 610C; Keithley Instruments, Cleveland, OH or model MEZ-8201; Nihon Kohden, Tokyo) using the standard technique, and recorded on a chart (model R-202 or R-002; Rikadenki, Tokyo) (20).

Measurement of net $\mathrm{Na}^{+}$reabsorption $(\mathrm{JNa})$ and $\mathrm{K}^{+}$secretion $(\mathrm{JK})$ : $\mathrm{Na}^{+}$and $\mathrm{K}^{+}$concentrations in the perfusate and collected fluid were measured by ultramicroflamephotometry using a microanalysis flame photometer (model AFA-707-D; Apel Co., Tokyo). JNa and JK were determined using the following equation:

$V_{\mathrm{o}}=V_{\mathrm{p}} / t$

$\mathrm{JNa}$ or $\mathrm{JK}(\mathrm{pEq} / \mathrm{mm}$ tubule $/ \mathrm{min})=\left(K_{\mathrm{i}}-K_{\mathrm{o}}\right) \cdot V_{\mathrm{o}} / L$,

where $V_{\mathrm{o}}$ is the collection rate, $V_{\mathrm{p}}$ and $t$ are the volume of the constriction pipette and the collection time, respectively, $K_{i}$ and $K_{o}$ are the concentrations of $\mathrm{Na}^{+}$or $\mathrm{K}^{+}$in the perfusate and collected fluid, respectively, and $L$ is the length of the tubule, which was measured directly by eyepiece reticule at the end of each experiment.

Measurement of osmotic water permeability: hydraulic conductivity $\left(L_{\mathrm{p}}\right)$ (centimeters per atmosphere per second), a parameter of lumen-to-bath osmotic water flow, was calculated as follows (1):

$L_{\mathrm{p}}=(1 / R T S) \cdot\left(1 / O_{\mathrm{b}}\right)^{2} \cdot\left[O_{\mathrm{b}} \cdot\left(V_{\mathrm{i}}-V_{\mathrm{o}}\right)\right.$

$\left.+O_{\mathrm{i}} \cdot V_{\mathrm{i}} \cdot \operatorname{Ln}\left\{\left(O_{\mathrm{b}}-O_{\mathrm{i}}\right) \cdot V_{\mathrm{i}} /\left(O_{\mathrm{b}} \cdot V_{\mathrm{o}}-O_{\mathrm{i}} \cdot V_{\mathrm{i}}\right)\right\}\right]$,

where $R$ is the gas constant, $T$ is the temperature of the bath medium (degree kelvin), $S$ is the luminal surface area of the tubule calculated from the tubule length and an assumed lumen diameter of $20 \mu \mathrm{m}, O_{\mathrm{b}}$ and $O_{\mathrm{i}}$ are the osmolalities of the bath medium and perfusate, respectively, and $V_{\mathrm{o}}$ and $V_{\mathrm{i}}$ are the collection rate and perfusion rate $\left(V_{\mathrm{i}}\right.$ $=V_{\mathrm{o}} \cdot C_{\mathrm{o}} / C_{\mathrm{i}}$, where $C_{\mathrm{o}}$ and $C_{\mathrm{i}}$ are radioactivities [counts per minute per nanoliter] of the perfusate, respectively, determined by a liquid scintillation counter [LSC-671; Aloka, Tokyo]).

As is well known (1), the hydroosmotic response of the rabbit CCD to AVP at $37^{\circ} \mathrm{C}$ is not stable. In our experiments, $L_{\mathrm{p}}$ increased from a basal level to a transient peak in 25-45 min after addition of AVP to the bath medium. Subsequently in some tubules, a small (10-20\% in average) decline in $L_{\mathrm{p}}$ due to an unknown mechanism followed, despite the presence of a constant concentration of AVP. Thus, the peak $L_{\mathrm{p}}$ induced by AVP was defined as the mean of the three largest values during exposure to AVP.

Reagents. Acetazolamide, isoproterenol, glucagon, and ouabain were purchased from Sigma Chemical Co., St. Louis, MO. AVP was purchased from Sigma Chemical Co., and Calbiochem Corp., San Diego, CA. $\left[{ }^{3} \mathrm{H}\right]$ Inulin was purchased from New England Nuclear, Boston, MA.

Statistics. Data are represented as mean \pm SE. Unless otherwise specified, one-way analysis of variance (ANOVA) was applied to determine statistical significance. The differences at $P<0.05$ were considered significant.

\section{Results}

Using isotonic perfusate, the effects of various doses of luminal AVP on $V_{t}$ were examined first. In these experiments where $V_{t}$

1. Abbreviations used in this paper: $L_{\mathrm{p}}$, hydraulic conductivity; $\mathrm{OMCDi}$, outer medullary collecting duct; $V_{t}$, transepithelial voltage. was measured exclusively, a high perfusion rate $(\sim 50 \mathrm{nl} / \mathrm{min})$ was chosen and the perfused fluid was collected in a large volume $(\sim 30 \mu \mathrm{l})$ pipette to avoid changes in perfusion rate and perfusion pressure that might affect $V_{t}$ during collection or exchange of perfusate. As shown in Figs. 1 and 2, $V_{t}$ remained unchanged after an exchange of perfusate to one containing vehicle alone or $10 \mathrm{pM}$ AVP. AVP at $100 \mathrm{pM}$ occasionally caused a small hyperpolarization, as shown in Fig. $1 A$, but this was not always observed, and the percentage change of $V_{t}$ caused by 10 or 100 pM AVP was not significantly different from zero. On the other hand, 1 or $10 \mathrm{nM}$ luminal AVP always induced hyperpolarization of $V_{\mathrm{t}}$. AVP at $100 \mathrm{nM}$ or $1 \mu \mathrm{M}$ did not further hyperpolarize $V_{t}$, as shown in Fig. 2. Thus, a subnanomolar concentration appeared to be the threshold concentration, and 1 and $10 \mathrm{nM}$ were the half-maximal and maximal concentrations required for the $V_{\mathrm{t}}$ change, respectively. The $V_{\mathrm{t}}$ was restored after the removal of luminal AVP (Fig. 1).

To further evaluate the specificity of this effect of luminal AVP, another polypeptide hormone, glucagon (10 nM), was applied to the lumen, but no change in $V_{t}$ was observed $(n=3$, data not shown). Also, when a different segment of the collecting duct, the inner stripe of the outer medullary collecting duct (OMCDi), was perfused $(n=3)$, neither $10 \mathrm{nM}$ luminal AVP nor $10 \mathrm{nM}$ luminal glucagon altered the $V_{t}$ (data not shown).

The hyperpolarization induced by luminal AVP was obviously distinct from that induced by basolateral AVP as shown in Fig. 3. Basolateral AVP caused a transient hyperpolarization, whereas a sustained hyperpolarization was induced by

A.

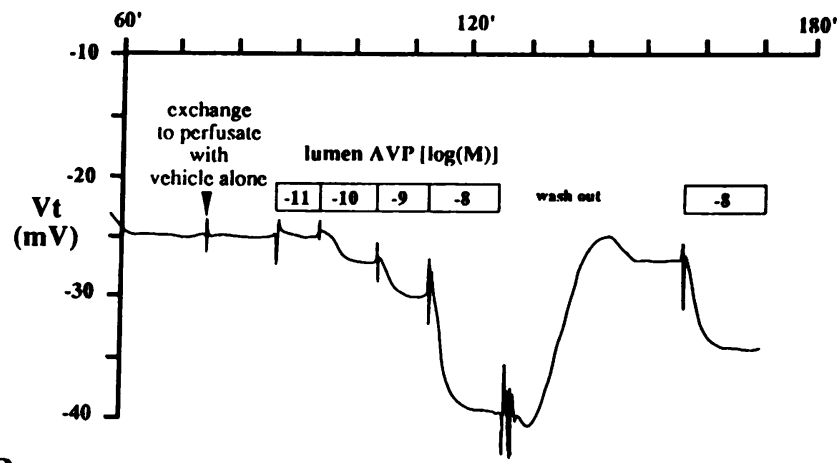

B.

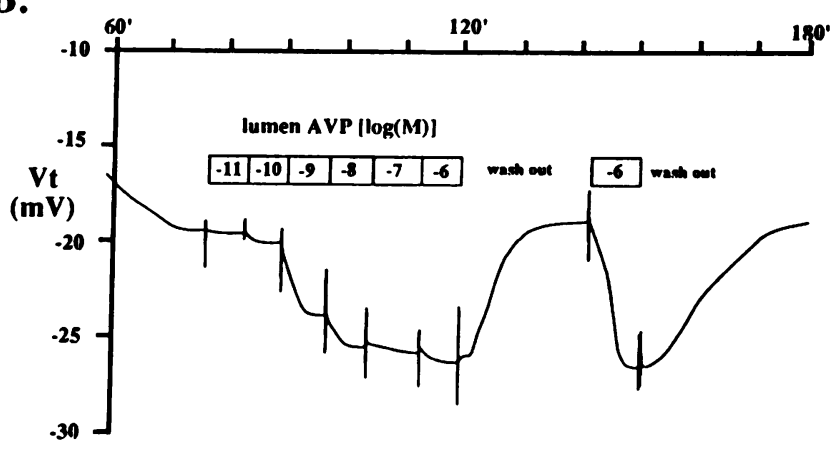

Figure 1. Effect of luminal AVP on $V_{\mathrm{t}}$ in the rabbit CCD perfused in vitro at $37^{\circ} \mathrm{C}$. Two original traces of $V_{t}$ are shown. AVPs from two different sources (Sigma Chemical Co. and Calbiochem Corp.) were used in $A$ and $B$, respectively. The maneuver to exchange luminal perfusate did not in itself alter $V_{t}$. Luminal AVP induced hyperpolarization in a dose-dependent manner in both $A$ and $B$. 


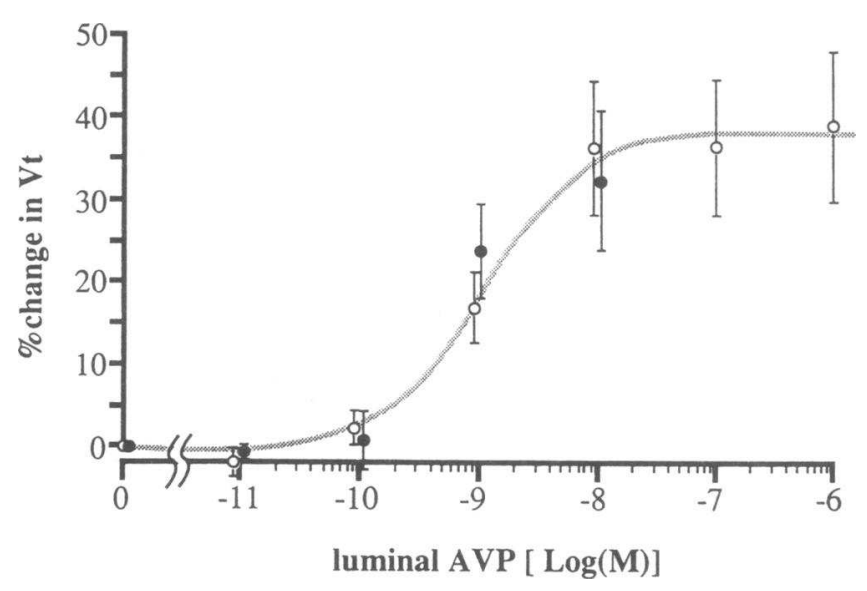

Figure 2. Dose dependency of luminal AVP induced hyperpolarization. Mean percentage $V_{t}$ were calculated from four to six experiments performed as shown in Fig. 1. Percentage $V_{t}$ was calculated as: $\left(V_{t}\right.$ basal $\left.V_{\mathrm{t}}\right) /$ basal $V_{\mathrm{t}} \times 100 \%$. No apparent discrepancy in dose dependency is seen between AVPs from two different sources. AVP from Sigma Chemical Co. (๑) or Calbiochem Corp. (o).

luminal AVP. The transient hyperpolarization induced by basolateral AVP started 1 to 2 min after addition of AVP to the bath medium reached a maximum lasting only several seconds within 5 to $8 \mathrm{~min}$ after addition of AVP, then $V_{\mathrm{t}}$ rapidly depolarized nearly to the basal $V_{t}$ level within $15 \mathrm{~min}$ (e.g., Fig. 3, $A$, $B, D$, and $E$ ). In the case of luminal AVP, hyperpolarization started 1 to $2 \mathrm{~min}$ after addition of AVP to the luminal perfusate, then maximal hyperpolarization was attained in 4 to 10 min. Subsequently, in about three-fourths of all tubules, the hyperpolarization was maintained for as long as luminal AVP was present (up to $30 \mathrm{~min}$ in flux studies presented later, e.g., Fig. $6 \mathrm{~A}$ ). In one-fourth of the tubules, after the 1- to 5-min hyperpolarization $V_{\mathrm{t}}$ was partially depolarized. This depolarization was slower than that after addition of basolateral AVP, and always stabilized at levels more negative than the basal $V_{\mathrm{t}}$ (e.g., Fig. $3 A$ ).

The $V_{t}$ change induced by AVP on one side of the CCD epithelium was not influenced by the presence of AVP on the contralateral side (Fig. 3, $A$ and $B$ ). Furthermore, as shown in Figs. $3 C-E$ and in Table $I$, the $V_{t}$ change induced by basolateral AVP was abolished in the presence of basolateral ouabain, a well known inhibitor of electrogenic $\mathrm{Na}^{+}$and $\mathrm{K}^{+}$transport in the $\operatorname{CCD}(21,22)$. In contrast, the $V_{\mathrm{t}}$ responses to luminal AVP and to bath isoproterenol, which is thought to alter $V_{t}$ via activating adenylyl cyclase exclusively in intercalated cells (23-26), were preserved.

In the study shown in Table $\mathrm{I}$, the $V_{\mathrm{t}}$ response to basolateral and luminal AVP was examined in the presence and absence of ouabain according to the protocol shown in Fig. $3 D$. By adding $10 \mu \mathrm{M}$ ouabain to the bath medium, the $V_{\mathrm{t}}$ changed rapidly from $-14.0 \pm 2.4$ to $7.3 \pm 1.4 \mathrm{mV}(n=8)$. In the presence of ouabain, $100 \mathrm{pM}$ or $10 \mathrm{nM}$ basolateral AVP (100 pM; tubules $2-5,10 \mathrm{nM}$; tubules 6 and 7 in Table I) failed to change the $V_{\mathrm{t}}$ (mean $\Delta V_{\mathrm{t}}=-0.3 \pm 0.3 \mathrm{mV}$, not significantly different from zero by $t$ test), whereas the $V_{\mathrm{t}}$ change induced by $10 \mathrm{nM}$ luminal AVP (tubules 1-8) was preserved (mean $\Delta V_{t}=-5.3 \pm 0.9$ $\mathrm{mV}$ ). In tubules 3-8, by removing ouabain and AVP from the bath medium and perfusate, $V_{\mathrm{t}}$ slowly became negative and stabilized at $-9.4 \pm 1.7 \mathrm{mV}$ in 60 to $90 \mathrm{~min}$. At this point, 100

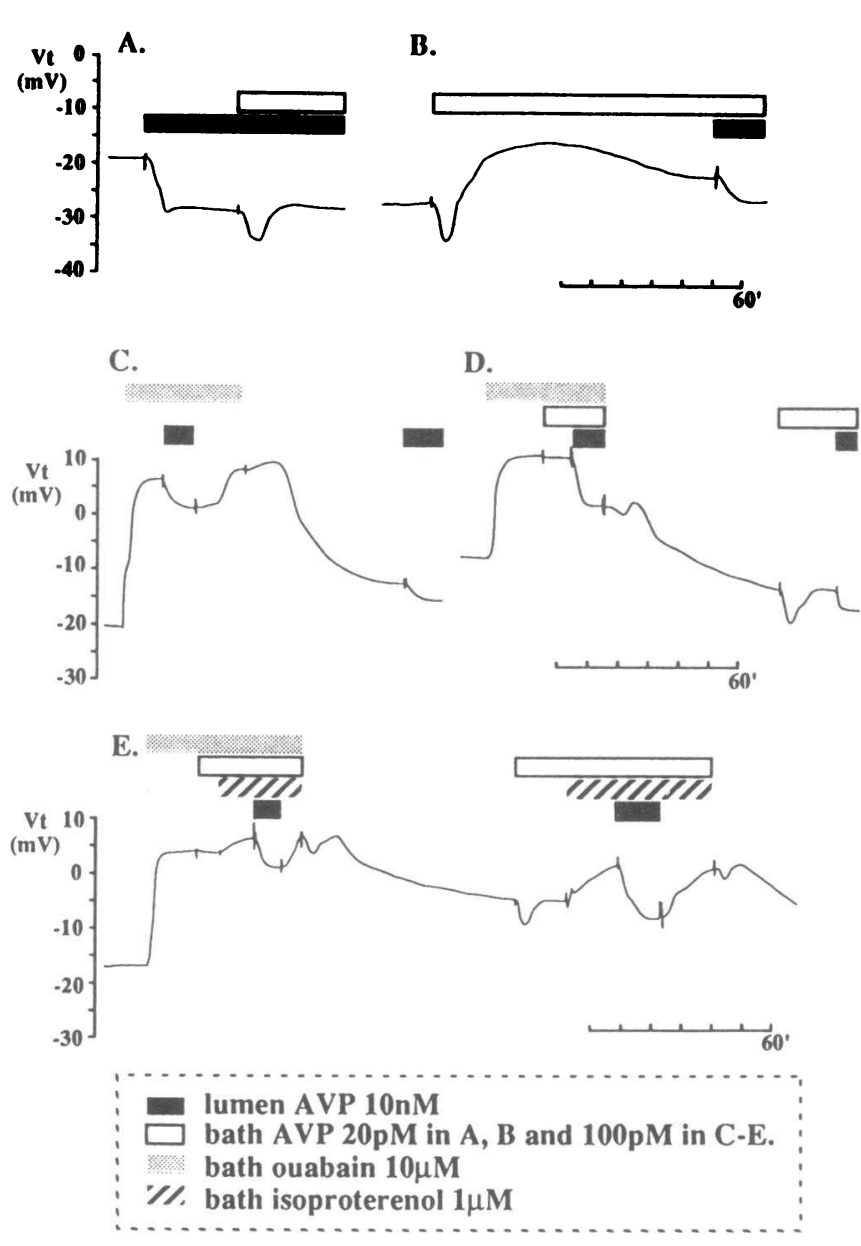

Figure 3. Comparison of the $V_{1}$ change induced by basolateral AVP ( $20 \mathrm{pM}$ in $A$ and $B, 100 \mathrm{pM}$ in $C-E$.) versus luminal AVP (10 nM). Original traces are shown. ( $A$ and $B$ ) Luminal AVP persistently hyperpolarizes $V_{t}$, whereas the hyperpolarization induced by basolateral AVP is transient $(A, B$, and the latter part of $D$ and $E)$. AVP pretreatment on one side of the tubular epithelium does not interfere with the $V_{\mathrm{t}}$ change induced by the contralateral AVP $(A$ and $B)$. The $V_{\mathrm{t}}$ changes induced by luminal AVP $(C-E)$ and basolateral isoproterenol $(E)$ are preserved in the presence of ouabain, whereas basolateral AVP fails to alter $V_{\mathrm{t}}$ when ouabain is present $(D$ and $E$ ).

pM basolateral AVP was rechallenged in tubules 3-7. In all of these five tubules, the $V_{\mathrm{t}}$ response to basolateral AVP, i.e., transient hyperpolarization, was restored (mean $\Delta V_{\mathrm{t}}=-4.0 \pm 0.9$ $\mathrm{mV}$ ). In tubules 4-8, $10 \mathrm{nM}$ luminal AVP was subsequently added, and this induced sustained hyperpolarization in all of the tubules (mean $\Delta V_{\mathrm{t}}=-4.2 \pm 1.0 \mathrm{mV}$ ).

Next, in a different group of tubules, net Na reabsorption and $\mathrm{K}$ excretion ( $\mathrm{JNa}$ and $\mathrm{JK}, \mathrm{pEq} / \mathrm{mm}$ tubule/min) were measured using the same isotonic perfusate (Fig. 4). To enhance the changes in perfusate $\mathrm{Na}^{+}$or $\mathrm{K}^{+}$concentration during perfusion, long tubules $(\sim 2.0 \mathrm{~mm})$ were perfused at a slow perfusion rate $(\sim 5 \mathrm{nl} / \mathrm{min})$. In these experiments $(n=6)$ also, luminal AVP caused a sustained hyperpolarization of $V_{\mathrm{t}}$ (from $-20.7 \pm 2.9$ to $-34.1 \pm 4.7 \mathrm{mV} ; P<0.01$ by paired $t$ test) at a constant perfusion rate (from $5.3 \pm 0.3$ to $5.0 \pm 0.3 \mathrm{nl} / \mathrm{min}$; NS by paired $t$ test). Interestingly, despite the evident change in $V_{t}$, $\mathrm{JNa}$ was stimulated only slightly (from $30.4 \pm 5.5$ to $32.7 \pm 4.7$ $\mathrm{pEq} / \mathrm{mm}$ per min; $P<0.025$ by paired $t$ test) and JK did not 
Table I. Differential Effect of Ouabain on Hyperpolarization of $V_{t}$ by Basolateral Versus Luminal AVP

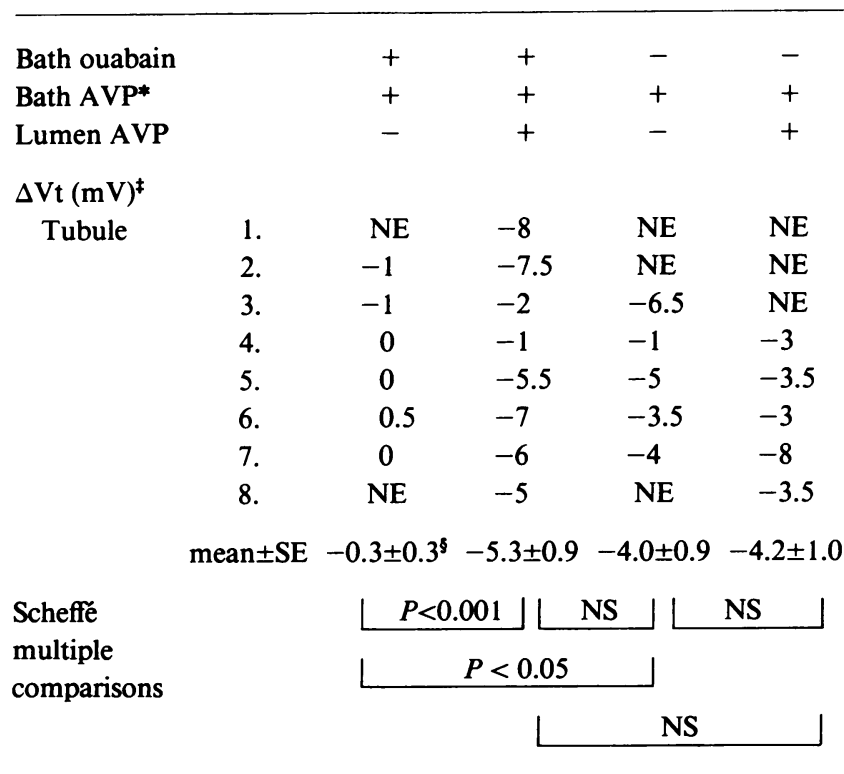

* $\Delta V_{\mathrm{t}}(\mathrm{mV})$ was defined as: $V_{\mathrm{t}}$ at maximum hyperpolarization during exposure to basolateral luminal AVP - Vt just before each AVP addition. ${ }^{\ddagger} 100 \mathrm{pM}$ in tubules $2-5,10 \mathrm{nM}$ in tubules 6 and 7 . ${ }^{8}$ Not significantly different from zero. NE, Not examined.

change significantly (from $28.5 \pm 1.7$ to $29.0 \pm 1.7 \mathrm{pEq} / \mathrm{mm}$ per min; NS by paired $t$ test).

To explore the mechanism of the luminal AVP-induced hyperpolarization further, we also employed acetazolamide, which is known to be an inhibitor of electrogenic $\mathrm{H}^{+}$secretion in the rabbit CCD (27). In these experiments, tubules were perfused at a high perfusion rate $(\sim 50 \mathrm{nl} / \mathrm{min})$, as in the doseresponse and ouabain studies. In the absence of acetazolamide, the $V_{\mathrm{t}}$ was hyperpolarized by $10 \mathrm{nM}$ luminal AVP from $-14.9 \pm 3.1$ to $-21.4 \pm 3.7 \mathrm{mV}$, which recovered to $-16.4 \pm 3.3$ within 20 to 30 min after AVP washout (Fig. 5 and Table II). Then in the same tubules, luminal AVP was rechallenged in
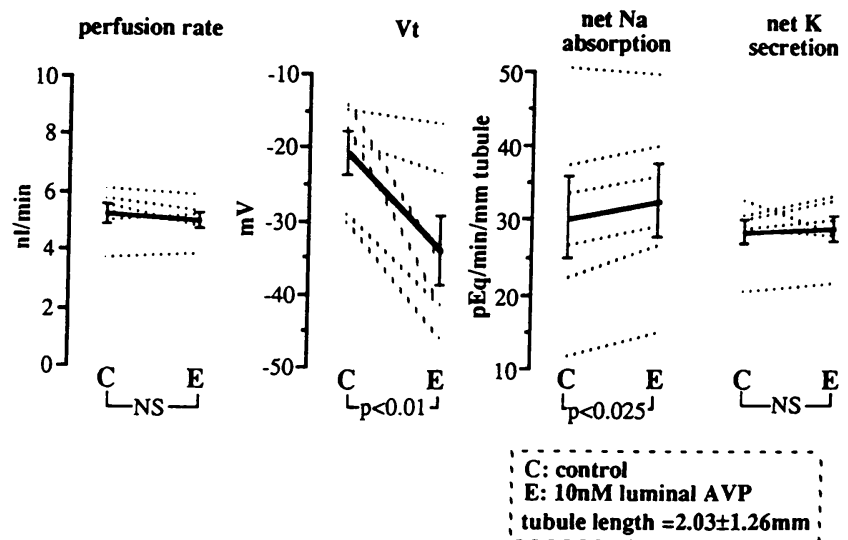

tubule length $=2.03 \pm 1.26 \mathrm{~mm}$

Figure 4. Effect of luminal AVP on net $\mathrm{Na}$ and $\mathrm{K}$ flux in CCD. Dotted line and solid line represent individual experiment and the mean, respectively. At a constant perfusion rate, luminal AVP hyperpolarizes $V_{\mathrm{t}}$ with negligible changes in the net $\mathrm{Na}$ and $\mathrm{K}$ fluxes. Statistical analyses were done by paired $t$ test.

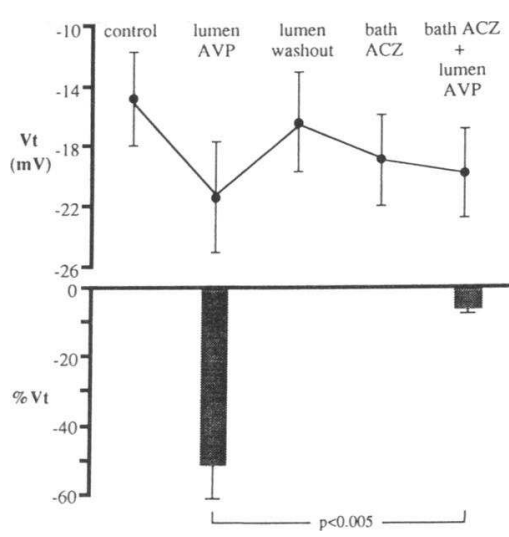

Figure 5. Effect of 200 $\mu \mathrm{M}$ bath acetazolamide $(A C Z)$ on $10 \mathrm{nM}$ luminal AVP-induced hyperpolarization. (See Table I for individual data.) The mean $V_{t}$ of five experiments is shown in the upper panel. The changes in $V$, between each period were significant when compared by paired $t$ test. The lower panel represents the magnitude of hyperpolarization by luminal AVP (percentage $V_{t}$ ) in the absence and in the presence of $\mathrm{ACZ}$.

the presence of $200 \mu \mathrm{M}$ acetazolamide in the bath. By addition of acetazolamide, $V_{\mathrm{t}}$ was initially hyperpolarized and partially reversed, stabilizing at $-18.9 \pm 3.0 \mathrm{mV}$ in $\sim 30 \mathrm{~min}$. Subsequent luminal AVP hyperpolarized $V_{t}$ only slightly to $-19.8 \pm 3.0 \mathrm{mV}$. Both $\Delta V_{\mathrm{t}}$ and percentage $V_{\mathrm{t}}$ changes were significantly smaller in the presence of acetazolamide (Fig. 5, Table II $A$ ).

To exclude the possibility that the attenuated hyperpolarization in response to the second luminal AVP was not due to acetazolamide, but resulted from desensitization of the tubule by the initial luminal AVP, we carried out another five timematched experiments, in which $10 \mathrm{nM}$ luminal AVP was applied twice with an interval of 40 to $60 \mathrm{~min}$ (Table II $B$ ). In this study, desensitization was denied since the $V_{t}$ responses to the first and second luminal AVP challenges were not different. Furthermore, because the hyperpolarization induced by acetazolamide itself might have reduced the potential of the tubules to hyperpolarize further in response to luminal AVP, another procedure to hyperpolarize the $V_{t}$, i.e., luminal addition of $\mathrm{Ba}^{++}$, was tested. $\mathrm{Ba}^{++}$is a well known $\mathrm{K}$ channel blocker and hyperpolarizes the $V_{\mathrm{t}}$ of the rabbit CCD by inhibiting apical $\mathrm{K}$ secretion (28). Indeed, as shown in Table II $C, 2 \mathrm{mM}$ luminal $\mathrm{BaCl}_{2}$ pretreatment hyperpolarized $V_{\mathrm{t}}$ from $-12.9 \pm 1.9$ to $-22.2 \pm 3.2 \mathrm{mV}$. Nevertheless, the $V_{\mathrm{t}}$ was further hyperpolarized to $-29.4 \pm 4.8 \mathrm{mV}$ by superimposing luminal AVP. Both $\Delta V_{\mathrm{t}}$ and percentage $V_{\mathrm{t}}$ changes induced by luminal AVP in the presence of $\mathrm{Ba}$ were not significantly different from those induced by luminal AVP in untreated tubules, as shown in Table II, $A$ and $C$. Thus, it was unlikely that hyperpolarization of $V_{\mathrm{t}}$ itself spoiled the $V_{t}$ change induced by luminal AVP in the acetazolamide study.

Finally, the effect of luminal AVP on osmotic water permeability in CCD was investigated (Fig. 6) in the presence of an osmotic gradient between the bath medium $(300 \mathrm{mOsmol})$ and the low $\mathrm{NaCl}$ hypotonic perfusate $(150 \mathrm{mOsmol})$. To stimulate water transport, $20 \mathrm{pM}$ basolateral AVP was chosen because this concentration is within the physiologic range for plasma AVP and exclusively stimulates the cyclic AMP system without considerably affecting noncyclic AMP-related signaling systems coupled to the so called $V_{1}$ type receptor (1). Also, this concentration is comparable to the plasma AVP concentration recorded in a previous human study ( $\sim 40 \mathrm{pM})$ when nanomolar ( $7 \mathrm{nM})$ AVP was measured in the urine (9). 
Table II. Effect of Acetazolamide (ACZ) on Luminal AVP-induced Hyperpolarization

\begin{tabular}{|c|c|c|c|c|c|c|c|c|}
\hline Vt of tubule & 1 & 2 & 3 & 4 & 5 & mean $V_{\mathbf{t}}$ & mean $\Delta V_{\mathrm{t}}$ & mean $\% V_{\mathrm{t}}$ \\
\hline \multicolumn{9}{|l|}{ A. Effect of bath acetazolamide } \\
\hline control & -15.5 & -19 & -9 & -7 & -24 & $-14.9 \pm 3.1$ & & \\
\hline lumen AVP $10 \mathrm{nM}$ & -22 & -24 & -17 & -11 & -33 & $-21.4 \pm 3.7$ & $-6.5 \pm 0.9$ & $-50.4 \pm 10.8$ \\
\hline lumen washout & -14 & -22 & -12 & -8 & -26 & $-16.4 \pm 3.3$ & & \\
\hline bath ACZ $200 \mu \mathrm{M}$ & -18.5 & -24 & -14 & -11 & -27 & $-18.9 \pm 3.0$ & $-2.5 \pm 0.6$ & $-19.8 \pm 6.5$ \\
\hline bath ACZ + lumen AVP & -20 & -24 & -15 & -11.5 & -28.5 & $-19.8 \pm 3.0$ & $-1.5 \pm 0.3^{*}$ & $-5.1 \pm 1.4^{*}$ \\
\hline \multicolumn{9}{|l|}{ B. Effect of repetitive lumen AVP } \\
\hline control & -20 & -8 & -16 & -7 & -10 & $-12.2 \pm 2.5$ & & \\
\hline lumen AVP $10 \mathrm{nM}$ (1st) & -27 & -13 & -20 & -11 & -15 & $-17.2 \pm 2.9$ & $-5.0 \pm 0.5$ & $-45.9 \pm 7.0$ \\
\hline lumen washout & -16 & -9 & -9 & -7 & -9.5 & $-10.1 \pm 1.5$ & & \\
\hline lumen AVP 10 nM (2nd) & -20 & -15 & -12 & -11 & -15.5 & $-14.7 \pm 1.6$ & $-4.6 \pm 0.6^{\ddagger}$ & $-49.1 \pm 8.4^{\ddagger}$ \\
\hline \multicolumn{9}{|l|}{ C. Effect of lumen $\mathrm{Ba}$} \\
\hline control & -11.5 & -9 & -11 & -13 & -20 & $-12.9 \pm 1.9$ & & \\
\hline lumen $\mathrm{Ba} 2 \mathrm{mM}$ & -25 & -17 & -15 & -21 & -33 & $-22.2 \pm 3.2$ & $-9.3 \pm 1.8^{8}$ & $-73.8 \pm 13.7^{8}$ \\
\hline lumen $\mathrm{Ba}+$ lumen AVP $10 \mathrm{nM}$ & -28 & -23 & -21 & -27 & -48 & $-29.4 \pm 4.8$ & $-7.2 \pm 2.0^{\prime \prime}$ & $-32.3 \pm 5.8^{\| \prime}$ \\
\hline lumen AVP washout & -24 & -19 & -18 & -21.5 & -31 & $-22.7 \pm 2.3$ & & \\
\hline
\end{tabular}

${ }^{*} P<0.005$ vs. lumen AVP alone. ${ }^{\ddagger}$ Not significantly different from first lumen AVP by paired $t$ test. ${ }^{8} P<0.01$ vs. bath ACZ in protocol A. " Not significantly different from lumen AVP in protocol A or those in protocol B by nonpaired $t$ test.

Eight tubules served as controls for the effect of $20 \mathrm{pM}$ basolateral AVP on $V_{\mathrm{t}}$ and $L_{\mathrm{p}}$ in the absence of luminal AVP, where $V_{\mathrm{t}}$ was transiently hyperpolarized from $-19.9 \pm 4.1$ to $-28.0 \pm 5.8 \mathrm{mV}$ (Fig. 6, upper panel), and $L_{\mathrm{p}}$ increased from a basal value of $5.9 \pm 1.5$ to a peak of $204.3 \pm 21.1 \times 10^{-7} \mathrm{~cm} / \mathrm{atm}$ per-s (Fig. 6, lower panel). In seven other tubules, even in the presence of a $\mathrm{NaCl}$ concentration gradient between the perfusate and bath medium, a rapid and sustained hyperpolarization of $V_{\mathrm{t}}$ from $-24.7 \pm 6.9$ to $-34.5 \pm 7.2 \mathrm{mV},(n=7, P<0.005$ by paired $t$ test) was observed upon luminal addition of $10 \mathrm{nM}$ AVP at a constant perfusion rate ( $V_{\mathrm{i}}$ : from $17.7 \pm 0.5$ to $17.0 \pm 0.6 \mathrm{nl} / \mathrm{min}$; NS by paired $t$ test), as shown in the upper panel of Fig. 6. In these tubules, $L_{\mathrm{p}}$ was not increased (from $3.9 \pm 0.8$ to $5.0 \pm 1.2 \times 10^{-7} \mathrm{~cm} /$ atm per $\mathrm{s}$; NS by paired $t$ test) by luminal AVP (Fig. 6, lower panel). Though subsequent addition of $20 \mathrm{pM}$ basolateral AVP further caused an intact transient hyperpolarization (Fig. $6 A ; \Delta V_{\mathrm{t}}$ change $=-8.9 \pm 2.3 \mathrm{vs}$. $-8.0 \pm 2.4 \mathrm{mV}$ in control experiments without luminal AVP; NS), the peak $L_{\mathrm{p}}$ induced by basolateral AVP was significantly lower in the presence of luminal AVP (Fig. 6, lower panel). Peak $L_{\mathrm{p}}$ was $134.9 \pm 19.2$ vs. $204.3 \pm 21.1 \times 10^{-7} \mathrm{~cm} / \mathrm{atm}$ per $\mathrm{s}$ in the control $(P<0.05)$.

\section{Discussion}

This study agrees with previous in vitro microperfusion studies in which $250 \mu \mathrm{U} / \mathrm{ml}(\sim 500 \mathrm{pM}) \mathrm{AVP}$ was luminally applied to the rabbit CCD (12): luminal AVP, even at a concentration as high as $10 \mathrm{nM}$, failed to increase water permeability in contrast to basolateral AVP. However, we found for the first time that luminal AVP in the nanomolar range hyperpolarized $V_{\mathrm{t}}$ and antagonized the hydroosmotic effect of basolateral AV.P. The action of luminal AVP was quite different from that of basolateral AVP, and cannot have been due to leakage of luminal AVP into the bath medium, because $(a)$ the sustained hyperpolarization was distinct from the transient hyperpolarization induced by basolateral AVP, $(b)$ luminal AVP did not interfere with the $V_{\mathrm{t}}$ response to basolateral AVP, and vice versa, $(c)$ basolateral ouabain abolished the $V_{t}$ response to basolateral AVP while the $V_{\mathrm{t}}$ change induced by luminal AVP was preserved, and $(d)$ no increase in $L_{\mathrm{p}}$ was induced by luminal AVP (basolateral AVP as low as $1 \mathrm{pM}$ is enough to stimulate $L_{\mathrm{p}}$ significantly in this system [1]). A nonspecific response to the perfusate exchange per se was also denied because luminal perfusion with vehicle only or with $10 \mathrm{nM}$ glucagon did not alter $V_{\mathrm{t}}$. In addition, parathyroid hormone $100 \mathrm{mU} / \mathrm{ml}$ has been previously reported not to alter $V_{t}$ in the rabbit CCD (29). Also, this $V_{t}$ response appears to be localized to the $C C D$, because OMCDi lacked any $V_{t}$ response to luminal AVP in this study, and the $V_{t}$ of the medullary thick ascending limb of loop of Henle of the rat was reportedly insensitive to luminal AVP at $\sim 5 \mathrm{nM}(30)$.

The $V_{\mathrm{t}}$ change induced by luminal AVP seems to be unique. In the light of detailed studies of the morphological and functional subtypes of CCD cells, where a variety of ion transport pathways have been identified and found to be hormonally regulated, the origin of $V_{t}$ in the CCD and the mechanism of its regulation cannot be explained simplistically without direct measurement of transport of various ions including $\mathrm{H}^{+}$, $\mathrm{Cl}^{-}, \mathrm{HCO}_{3^{-}}, \mathrm{Ca}^{++}, \mathrm{PO}_{4^{3-}}$, and $\mathrm{Mg}^{++}(31-34)$ in the apical and basolateral membranes of both principal and intercalated cells. However, because the principal cell is thought to participate, if not exclusively, in AVP-regulated water transport in the CCD $(26,35)$, the inhibition of the hydroosmotic effect of basolateral AVP by luminal AVP suggests that luminal AVP might alter $V_{\mathrm{t}}$ by directly or indirectly affecting ion transport in principal cells. Nevertheless, the $V_{t}$ change induced by luminal AVP is obviously distinct from that induced by basolateral AVP, and cannot be explained only by trivial changes in net $\mathrm{Na}$ or $\mathrm{K}$ transport. In the CCD, the principal cell is considered to be 

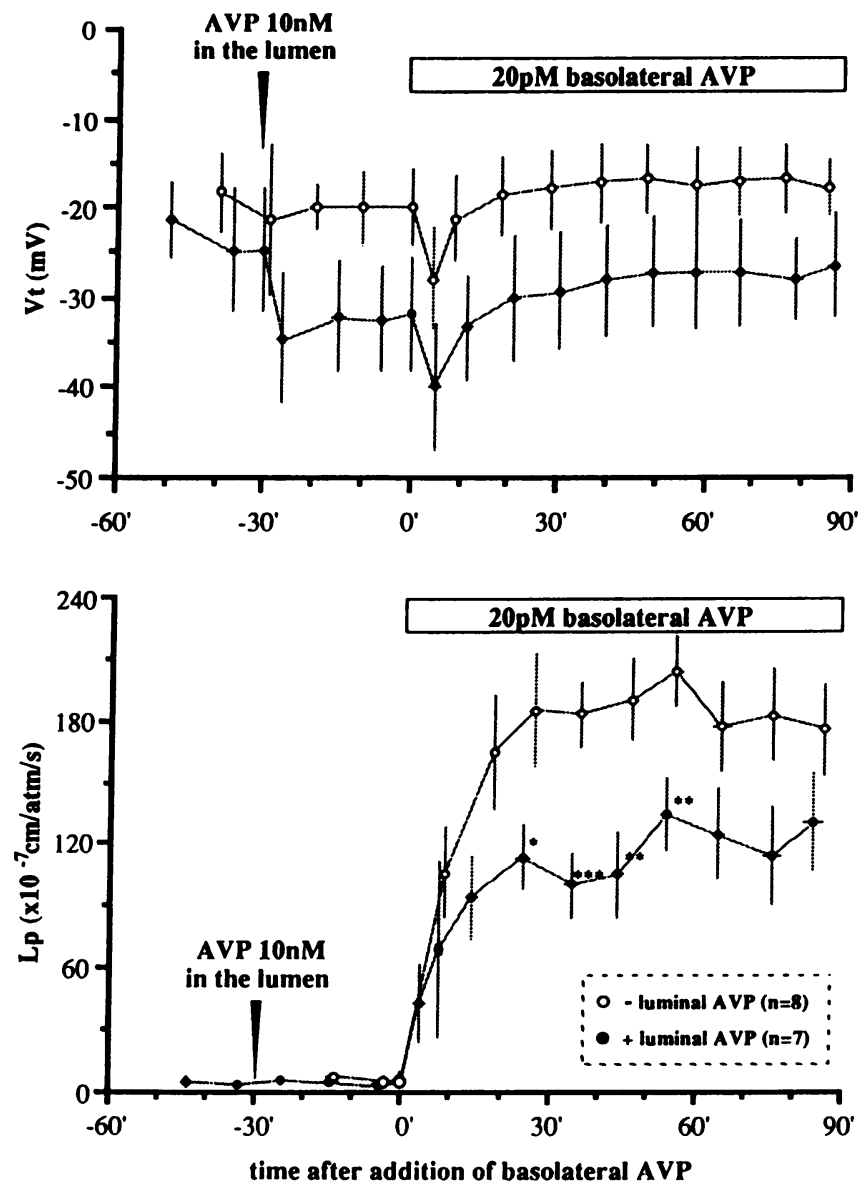

Figure 6. Effect of luminal AVP on water transport in CCD. The time courses of the mean $V_{\mathrm{t}}$ and $L_{\mathrm{p}}$ changes in control and experimental groups are shown in parallel. In the experimental group represented by solid circles, luminal AVP was added at $-30 \mathrm{~min}$. The upper panel shows sustained hyperpolarization by luminal AVP versus transient hyperpolarization by basolateral AVP. In the lower panel, while luminal administration of AVP itself has no significant effect on $L_{p}$, the increase in $L_{\mathrm{p}}$ induced by subsequent basolateral AVP is significantly attenuated compared with the control group during 20 to 50 min after addition of basolateral $\operatorname{AVP}\left({ }^{*} P<0.05,{ }^{* *} P<0.025,{ }^{* * *} P\right.$ $<0.005$ vs. corresponding control without luminal AVP). The peak $L_{\mathrm{p}}$ levels, defined in Methods, were also significantly different (see text).

primarily responsible for electrogenic $\mathrm{Na}^{+}$and $\mathrm{K}^{+}$transport. The driving force for transport of these cations in principal cells is provided by the basolateral ouabain-sensitive $\mathrm{Na}^{+} / \mathrm{K}^{+}$pump $(21,22,26)$. Thus, if luminal AVP hyperpolarizes $V_{t}$ by modulating $\mathrm{Na}$ or $\mathrm{K}$ transport in the principal cell, the $V_{\mathrm{t}}$ change induced by luminal AVP as well as the change caused by basolateral AVP should be suppressed by ouabain $(31,36-38)$. However, the $V_{\mathrm{t}}$ response was preserved in the presence of ouabain (Fig. 3, $C-E$, Table I), in accord with the lack of considerable change in net $\mathrm{Na}$ or $\mathrm{K}$ transport (Fig. 5). Furthermore, luminal $\mathrm{Ba}^{++}$did not inhibit the $V_{\mathrm{t}}$ change induced by luminal AVP (Table II C). This is also compatible with the lack of change in net $K$ transport and the inability of ouabain to inhibit the effect of luminal AVP on $V_{t}$. Taken together, the evidence is fairly convincing that $\mathrm{Na}$ and $\mathrm{K}$ transport in principal cells is not the crucial factor for the $V_{t}$ response to luminal AVP.
The effect of basolateral isoproterenol, which is thought to affect intercalated cell function preferentially (23-26), on $V_{t}$ was preserved in the presence of ouabain (Fig. $3 E$ ) in agreement with studies by others (23). Therefore, luminal AVP might hyperpolarize the $V_{\mathrm{t}}$ by modulating the transport of ions in intercalated cells. In this regard, the inhibitory effect of acetazolamide on the luminal AVP-induced hyperpolarization (Fig. 5, Table II $A$ ) is important. Electrogenic luminal acidification in the $C C D$, presumably via the apical $\mathrm{H}^{+}$pump of intercalated cells, is thought to play a role in shifting $V_{\mathrm{t}}$ in a positive direction, and acetazolamide has been found to inhibit this electrogenic acidification mechanism $(27,39)$. Studies by Koeppen and Helman (27) demonstrated that in the rabbit $\mathrm{CCD}$, by attenuating luminal acidification, acetazolamide can shift the $V_{t}$ in a negative direction in the presence of basolateral ouabain or in the absence of luminal $\mathrm{Na}^{+}$, both of which induce a lumen-positive $V_{\mathrm{t}}$ by abolishing $\mathrm{Na}^{+}$reabsorption. In our experiments, acetazolamide by itself slightly hyperpolarized $V_{\mathrm{t}}$, and significantly inhibited the luminal AVP-induced hyperpolarization (Fig. 5, Table II $A$ ). Our additional experiments shown in Table II $B$ and $C$ denied that this inhibition by acetazolamide was nonspecific. Thus, luminal AVP is suggested to induce hyperpolarization by inhibiting acetazolamide-sensitive electrogenic transport. Obviously, inhibition of apical $\mathrm{H}^{+}$ secretion is one of the most plausible mechanisms. However, this assumption needs to be confirmed by direct measurement of luminal acidification, because the effect of luminal AVP and its suppression by acetazolamide could be secondary to an alteration of intracellular $\mathrm{pH}$, which is known to modify $V_{\mathrm{t}}(40)$.

If inhibition of $\mathrm{H}^{+}$secretion is the common mechanism responsible for hyperpolarizations induced by acetazolamide and luminal AVP, why did the former induce a smaller hyperpolarization than the latter? Although we have no direct evidence to explain this difference, one possibility is that the hyperpolarization caused by acetazolamide could be hampered by concomitant inhibition of $\mathrm{Na}^{+}$reabsorption. Both Stoner et al. (39), and Koeppen and Helman (27) observed that the effect of acetazolamide in increasing the negativity of $V_{t}$ became overt if $\mathrm{Na}^{+}$reabsorption was inhibited previously by ouabain, a Nafree perfusate, or luminal amiloride. In contrast, because luminal AVP did not inhibit $\mathrm{Na}^{+}$reabsorption (Fig. 4), the hyperpolarization induced by luminal AVP would not be mitigated.

Also, in some studies $(27,41)$, basolateral ouabain per se has been found to suppress luminal acidification in the CCD. If this suppression is due to the same mechanism as that induced by acetazolamide, ouabain might also suppress luminal AVPinduced hyperpolarization. However, this was not obvious in our study (Table I), probably because of a difference in the mechanism or potency of these two agents in inhibiting acidification. Indeed Koeppen and Helman did not observe inhibition of acidification by ouabain in the absence of extracellular $\mathrm{Na}$ and $\mathrm{K}$, whereas that induced by acetazolamide was independent of these extracellular cations (27).

The mechanism of inhibition of the hydroosmotic effect of basolateral AVP by luminal AVP has also been unexplored. At least one previous study, in which no AVP-sensitive adenylyl cyclase was detected in the luminal membrane fraction of the bovine collecting duct, appears to argue against the possibility that the inhibition is related to an increase in cyclic AMP by luminal AVP (42). As mentioned earlier, nanomolar AVP is capable of autoinhibiting the hydroosmotic effect through activation of protein kinase $C$, elevation of the intracellular free 
$\mathrm{Ca}^{++}$concentration, or stimulation of prostaglandin synthesis (1). If certain AVP receptors coupled to these machineries reside in the apical membrane, luminal AVP might inhibit the hydroosmotic effect of basolateral AVP. In this regard, it is noteworthy that apical AVP has been found to stimulate prostaglandin synthesis in canine CCD cells in primary culture (15). However, all three of these mechanisms have been found to depolarize $V_{t}$ when activated. The protein kinase $\mathrm{C}$ activators phorbol myristate acetate and dioctanoylglycerol, the $\mathrm{Ca}$ ionophores A23187 and ionomycin, and prostaglandin $E_{2}$ rapidly depolarize the $V_{\mathrm{t}}$ of CCD when applied from the basolateral side (5-7, 18, and our unpublished data). In addition, all of these three signals are known to inhibit transepithelial Na reabsorption (5-7) in the CCD. Thus, it seems unlikely that these autoregulation mechanisms are employed by luminal AVP to inhibit water transport without either depolarizing the $V_{t}$ or inhibiting Na transport. However, we cannot rule out the possibility that luminal AVP selectively or locally activates these signals in the apical membrane or subapical cytoplasm, modulating cell functions in fashions different from those mentioned above.

The inhibition of the hydroosmotic effect by luminal AVP may be secondary to a change in electrolyte transport. The change in electrolyte transport underlying the luminal AVP-induced sustained hyperpolarization would alter the electrolyte composition or $\mathrm{pH}$ of the luminal perfusate as well as that of the intracellular fluid. Previous studies on amphibian urinary bladder and mammalian $\operatorname{CCD}(40,43-45)$ have shown that the hydroosmotic effect of basolateral AVP is sensitive to alteration in extracellular and/or intracellular $\mathrm{pH}$ and electrolyte composition. Such inhibitory mechanisms may function in either of the two cell types in the CCD, because the osmotic water permeability of intercalated cells as well as that of principal cells is reported to be increased in response to basolateral AVP $(26,35)$.

Finally, it also remains to be explored how AVP interacts with the luminal surface of the CCD. Although regulation of tubular epithelial functions by hormones in the lumen (urine) has been largely unexplored, a few hormones or biologically active substances are known or are suggested to act from the apical side of the renal tubule. For example, dopamine receptors have been found on the apical membrane of the proximal tubule (46). In the rabbit medullary thick ascending limb of loop of Henle, luminal prostaglandin $E_{2}$ has been found to depolarize $V_{t}(47)$. Also, it has been found that an apical $G$ protein linked to an ion channel exits in the rat medullary collecting duct (48). In the light of a previous study by Ando et al. (1), the higher threshold concentration for the action of luminal AVP compared with that of basolateral AVP and the antagonistic action of luminal AVP on water transport imply that a $V_{1}$-type receptor might reside on the apical membrane. However, studies should be undertaken with caution before classifying the luminal receptor for AVP, if present, as either the $V_{1}$ or $V_{2}$ type, because the interaction of AVP and CCD on the luminal surface may not be mediated by previously defined AVP receptors for signal transduction. Also, cross-reaction of AVP and oxytocin receptors should be considered (49). Furthermore, an early in vivo clearance study suggested that AVP filtered or excreted into urine might interact with catabolic enzymes on the luminal surface of the distal nephron segment (50). Such an interaction of the apical membrane of CCD cells and AVP for digestion might alter CCD functions secondarily.
Regardless of the mechanisms underlying the luminal action of AVP, an intriguing hypothesis is deducible. The concentration of urinary AVP would not be as high as the nanomolar range to exert its effect in the CCD under euhydration or mild dehydration (9-11). However, among the numerous factors and conditions that affect urine production in vivo, some might attenuate the antidiuretic action of basolateral AVP by increasing the urinary excretion of AVP. This inference seems reasonable to explain a paradox between the release of AVP and the local action of AVP in the kidney. For example, prostaglandins, kinins, and catecholamines are well known inhibitors of the antidiuretic action of AVP in the kidney $(17,19,25,47)$. However, they also stimulate the release of AVP from the neurohypophysis (8). Thus, under circumstances where these "renal" AVP antagonists are systemically increased, e.g., physical stress, excessive water reabsorption in the CCD due to elevated circulating AVP may be mitigated not only by the actions of these antagonists in the kidney, but also, in part, by the drastically increased AVP in the tubular lumen. In SIADH (syndrome of inappropriate secretion of antidiuretic hormone), a high luminal AVP concentration might also play a role in facilitating urine output, despite high levels of plasma AVP.

In conclusion, this study has demonstrated that luminal (urinary) AVP in the CCD modulates water and transepithelial voltage distinctly from basolateral (plasma) AVP. It is suggested that urine production is modulated by AVP at the CCD epithelium amphilaterally in vivo. The mechanisms as well as and the physiological significance of these actions of luminal AVP awaits further elucidation.

\section{Acknowledgments}

We thank Ms. Yoriko Kawahara for her expert assistance in experiments.

\section{References}

1. Ando, Y., M. D. Breyer, and H. R. Jacobson. 1989. Dose-dependent heterogeneous actions of vasopressin in rabbit cortical collecting ducts. Am. J. Physiol. 256:F556-F562.

2. Burnatowaska-Hledin, M. A., and W. S. Spielman. 1989. Vasopressin V, receptors on the principal cells of the rabbit cortical collecting tubule. J. Clin. Invest. 83:84-89.

3. Ando, Y., H. R. Jacobson, and M. D. Breyer. 1987. Phorbol myristate acetate, dioctanoylglycerol, and phosphatidic acid inhibit the hydroosmotic effect of vasopressin on rabbit cortical collecting tubule. J. Clin. Invest. 80:590-593.

4. Ando, Y., H. R. Jacobson, and M. D. Breyer. 1988. Phorbol ester and A23187 have additive but mechanistically separate effects on vasopressin action in rabbit cortical collecting tubule. J. Clin. Invest. 81:1578-1584.

5. Hays, S. R., M. Baum, and J. P. Kokko. 1987. Effects of protein kinase C activation on sodium, potassium, chloride, and total $\mathrm{CO}_{2}$ transport in the rabbit cortical collecting tubule. J. Clin. Invest. 80:1561-1570.

6. Stokes, J. B., and J. P. Kokko. 1977. Inhibition of sodium transport by prostaglandin $\mathrm{E}_{2}$ across the isolated, perfused rabbit collecting tubule. J. Clin. Invest. 59:1099-1104.

7. Palmer, L. G., and G. Frindt. 1987. Ca ionophore and phorbol ester inhibit Na channels in rat cortical collecting tubules. Fed. Proc. 46:495a. (Abstr.)

8. Robertson, G. L. 1985. Regulation of vasopressin secretion. In The Kidney: Physiology and Pathophysiology. D. W. Seldin and G. Giebisch editors. Raven Press, New York. 869-884.

9. Moses, A., and E. Steciak. 1986. Urinary and metabolic clearances of anginine vasopressin in normal subjects. Am. J. Physiol 251:R365-R370.

10. Kimura, T., and L. Share. 1981. Characterization of the renal handling of vasopressin in the dog by stop-flow analysis. Endocrinology. 109:2089-2094.

11. Rabkin, R., L. Share, P. A. Payne, J. Young, and J. Crofton. 1979. The handling of immunoreactive vasopressin by the isolated perfused rat kidney. $J$. Clin. Invest. 63:6-13. 
12. Grantham, J. J., and M. B. Burg. 1966. Effect of vasopressin and cyclic AMP on permeability of isolated collecting tubules. Am. J. Physiol. 211:255-259.

13. Orloff, J., and J. S. Handler. 1962. The similarity of effects of vasopressin adenosine-3',5'-phosphate (cyclic AMP) and theophylline on the toad bladder. $J$. Clin. Invest. 41:702-709.

14. Leaf, A., J. Anderson, and L. B. Page. 1958. Active sodium transport by the isolated toad bladder. J. Gen. Physiol. 41:657-668.

15. Garcia-Perez, A., and W. L. Smith. 1984. Apical-basolateral membrane asymmetry in canine cortical collecting tubule cells. J. Clin. Invest. 74:63-74.

16. Tomita, K., J. J. Pisano, and M. A. Knepper. 1985. Control of sodium and potassium transport in the cortical collecting duct of the rat. J. Clin. Invest. 76:132-136.

17. Stokes, J. B. 1985. Modulation of vasopressin-induced water permeability of the cortical collecting tubule by endogenous and exogenous prostaglandins. Mineral Electrolyte Metab. 11:240-248.

18. lino, Y., and M. Imai. 1978. Effects of prostaglandins on Na transport in isolated collecting tubules. Pflügers Arch. 373:125-132.

19. Schuster, V. L., J. P. Kokko, and H. R. Jacobson. 1984. Interactions of lysyl-bradykinin and antidiuretic hormone in the rabbit cortical collecting tubule. J. Clin. Invest. 73:1659-1667.

20. Ando, Y., K. Tabei, H. Furuya, and Y. Asano. 1989. Glucagon stimulates chloride transport independently of cyclic AMP in the rat medullary thick ascending limb. Kidney Int. 36:760-767.

21. Grantham, J. J., M. B. Burg, and J. Orloff. 1970. The nature of transtubular $\mathrm{Na}$ and $\mathrm{K}$ transport in isolated rabbit renal collecting tubules. J. Clin. Invest. 49:1815-1826.

22. Wingo, C. S. 1989. Active proton secretion and potassium absorption in the rabbit outer medullary collecting duct: functional evidence for proton-potassium-activated adenosine triphosphatase. J. Clin. Invest. 84:361-365.

23. Iino, Y., J. L. Troy, and B. M. Brenner. 1981. Effects of catecholamines on electrolyte transport in cortical collecting tubule. J. Membr. Biol. 61:67-73.

24. Kimmel, P. L., and S. Goldfarb. 1984. Effects of isoproterenol on potassium secretion by the cortical collecting tubule. Am. J. Physiol. 246:F804-F810.

25. Krothapalli, R. K., W. B. Duffy, H. O. Senekjian, and W. N. Suki. 1983. Modulation of the hydroosmotic effect of vasopressin on the rabbit cortical collecting tubule by adrenergic agents. J. Clin. Invest. 72:287-294.

26. Fejes-Tóth, G., and A. Náray-Fejes-Tóth. 1989. Isolated principal and intercalated cells: hormone responsiveness and $\mathrm{Na}^{+}-\mathrm{K}^{+}$-ATPase activity. Am. J. Physiol. 256:F742-F750.

27. Koeppen, B. M., and S. I. Helman. 1982. Acidification of luminal fluid by the rabbit cortical collecting tubule perfused in vitro. Am. J. Physiol. 242:F521F531.

28. Frindt, G., and L. G. Palmer. 1987. Ca-activated $\mathrm{K}$ channels in apical membrane of mammalian CCT, and their role in $\mathrm{K}$ secretion. Am. J. Physiol. 252:F458-F467.

29. Shareghi, G. R., and L. C. Stoner. 1978. Calcium transport across segments of the rabbit distal nephron in vitro. Am. J. Physiol. 235:F367-F375.

30. Sasaki, S., and M. Imai. 1980. Effects of vasopressin on water and $\mathrm{NaCl}$ transport across the in vitro perfused medullary thick ascending limb of Henle's loop of mouse, rat, and rabbit kidney. Pflügers Arch. 383:215-221.
31. Stokes, J. B. 1982. Ion transport by the cortical and outer medullary collecting tubule. Kidney Int. 22:473-484.

32. Madsen, K., and C. C. Tisher. 1986. Structural-functional relationships along the distal nephron. Am. J. Physiol. 250:F1-F15.

33. Schuster, V. L. 1987. Chloride transport by the cortical and outer medullary collecting duct. Am. J. Physiol. 253:F203-F212.

34. Holt, W. F., and C. Lechene. 1981. ADH-PGE2 interactions in cortical collecting tubule. II. Inhibition of $\mathrm{Ca}$ and $\mathrm{P}$ reabsorption. Am. J. Physiol. 241:F461-F467.

35. Kirk, K. L. 1988. Origin of ADH-induced vacuoles in rabbit cortical collecting tubule. Am. J. Physiol. 254:F719-F733.

36. Stokes, J. B. 1981. Potassium secretion by cortical collecting tubule: relation to sodium absorption, luminal sodium concentration, and transepithelial voltage. Am. J. Physiol. 241:F395-F402.

37. Frindt, G., and M. B. Burg. 1972. Effect of vasopressin on sodium transport in renal cortical collecting tubules. Kidney Int. 1:224-231.

38. Holt, W. F., and C. Lechene. 1981. ADH-PGE ${ }_{2}$ interactions in cortical collecting tubule. I. Depression of sodium transport. Am. J. Physiol. 241:F452F460.

39. Stoner, L. C., M. B. Burg, and J. Orloff. 1974. Ion transport in cortical collecting tubule; effect of amiloride. Am. J. Physiol. 227:453-459.

40. Hamm, L. L., C. Gillespie, and S. Klahr. 1985. NH4Cl inhibition of transport in the rabbit cortical collecting tubule. Am. J. Physiol. 248:F631-F637.

41. Laski, M. E. 1987. Total CO2 flux in isolated collecting tubules during carbonic anhydrase inhibition. Am. J. Physiol. 252:F322-F330.

42. Schwartz, I. L., L. J. Shaltz, E. Kinne-Saffran, and R. Kinne. 1974. Target cell polarity and membrane phosphorylation in relation to the mechanism of action of antidiuretic hormone. Proc. Natl. Acad. Sci. USA. 71:2595-2599.

43. Brem, A. S., E. Eich, M. Pearl, and A. Taylor. 1985. Anion transport inhibitors: effect on water and sodium transport in the toad urinary bladder. Am. J. Physiol. 248:F594-F601.

44. Lorenzen, M., A. Taylor, and E. E. Windhager. 1983. pH effect on osmotic response of collecting tubules to vasopressin and 8-CPT-cAMP. Am. J. Physiol. 245:F188-F197.

45. Parisi, M., J. Chevalier, and J. Bourguet. 1979. Influence of mucosal and serosal $\mathrm{pH}$ on antidiuretic action in frog urinary bladder. Am. J. Physiol. 237:F483-F486.

46. Felder, C. C., A. M. McKelvey, M. S. Gitler, G. M. Eisner, and P. A. Jose 1989. Dopamine receptor subtypes in renal brush border and basolateral membranes. Kidney Int. 36:183-193.

47. Stokes, J. B. 1979. Effect of prostaglandin $E_{2}$ on chloride transport across the rabbit thick ascending limb of Henle. J. Clin. Invest. 64:495-502.

48. Light, D. B., D. A. Ausiello, and B. M. Stanton. 1989. Guanine nucleotide-binding protein, $\alpha i-3$, directly activates a cation channel in rat renal inner medullary collecting duct cells. J. Clin. Invest. 84:352-356.

49. Tribollet, E., C. Barberis, J. Dreifuss, and S. Jard. 1988. Autoradiographic localization of vasopressin and oxytocin binding sites in rat kidney. Kidney Int. 33:959-965.

50. Walter, R., and R. H. Bowman. 1973. Mechanism of inactivation of vasopressin and oxytocin by the isolated perfused rat kidney. Endocrinology. 92:189-193. 